\title{
Brief Facts about COVID-19 (SARS-CoV-2)
}

\author{
Sorush Niknamian \\ MD PhD Dr.PH, Military Medicine Department, Liberty University, USA \\ Member of International Society of Infectious Diseases
}

Asian Journal of Complementary and Alternative Medicine. Volume 09 Issue 1

Published on: 12/01/2021

*Author for Correspondence: Sorush Niknamian, MD PhD Dr.PH, Military Medicine Department, Liberty University, USA; Email: so.niknamian@gmail.com.

Cite this article as: Niknamian S. Brief Facts about COVID-19 (SARS-CoV-2). Asian Journal of Complementary and Alternative Medicine, Vol 9(1), 1-5:2021.

\begin{abstract}
Coronaviruses are a group of related viruses that cause diseases in mammals and birds. In humans, coronaviruses cause respiratory tract infections that can range from mild to lethal. Mild illnesses include some cases of the common cold, while more lethal varieties can cause SARS, MERS, and COVID-19. The outbreak was identified in Wuhan, China, in December 2019, declared to be a Public Health Emergency of International Concern on 30 January 2020, and recognized as a pandemic on 11 March 2020. Coronaviruses are the subfamily Orthocoronavirinae, within the family of Coronaviridae, order Nidovirales, and realm Riboviria. They are enveloped viruses with a positive-sense single-stranded RNA genome and a nucleocapsid of helical symmetry. The genome size of coronaviruses is approximately from 26 to 32 kilobases. Coronaviruses were first discovered in the 1930s and Human coronaviruses were discovered in the 1960s. The earliest ones studied were from human patients with the common cold, which were later named human coronavirus $229 \mathrm{E}$ and human coronavirus OC43. Other human coronaviruses have since been identified, including SARS-CoV in 2003, HCoV NL63 in 2004, HKU1 in 2005, MERS-CoV in 2012, and SARS-CoV-2 in 2019. Most of these have involved serious respiratory tract infections
\end{abstract}

\section{INTRODUCTION}

\section{COVID-19}

COVID-19 is caused by the newly discovered coronavirus SARS CoV-2, previously called 2019-nCoV. It belongs to the Coronaviridae family and is broadly distributed in humans and other mammals. hCoV-229E, OC43, NL63 and HKU1 are some of the known coronaviruses that cause mild respiratory diseases unlike SAR-CoV and MERS that cause severe to fatal respiratory diseases [1].

Why is SARS-CoV-2 spreading faster than its two ancestors? Why is SARS-CoV-2 lethal? Recent publications have shown that there are differences in their genome structure and immunological response to SARS-CoV-2 infection. The key markers involved in these interactions include Spike protein (S), Nucleocapsid (N), ACE-2 receptor, FURIN protease in addition to the cytokines.

Spike Protein (S): SARS-CoV2 enters the cells through the Spike mediated interaction with the ECD domain of the ACE2 cell receptor. A recombinant fusion protein (RBD of Spike protein and ECD of membrane protein) can a great tool to investigate this interaction (Figure 1).

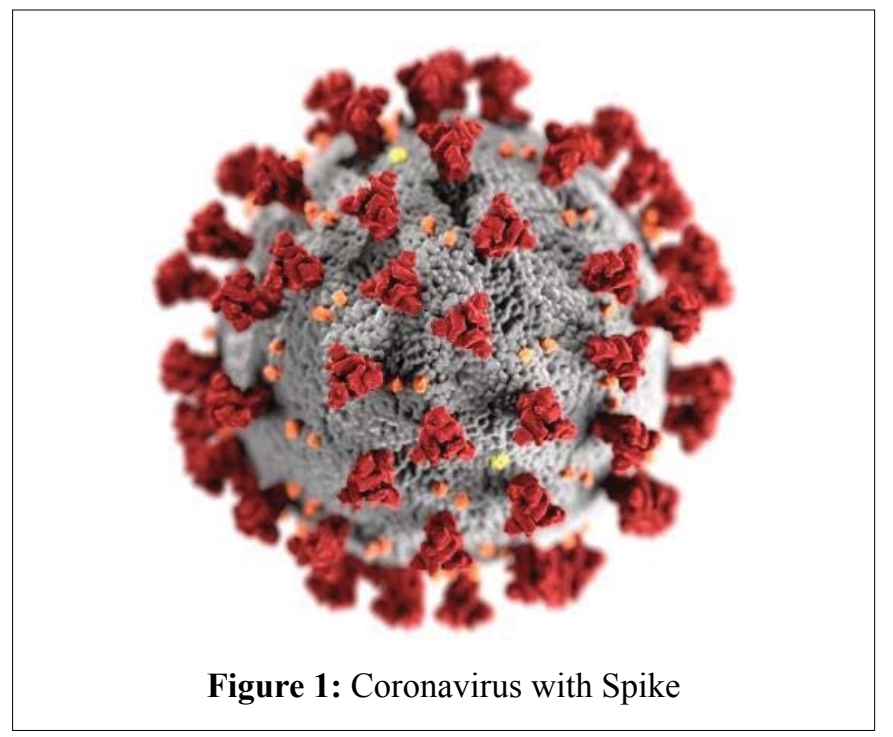

Transmembrane Serine Protease 2 (TMPRSS2): This is a serine protease that cleaves and activates the viral spike glycoproteins which facilitates virus-cell membrane fusions. A recent study showed that SARS-CoV-2 needs both ACE2 receptor and serine protease TMPRSS2 for protein priming to enter the cell [2] (Figure 2). 


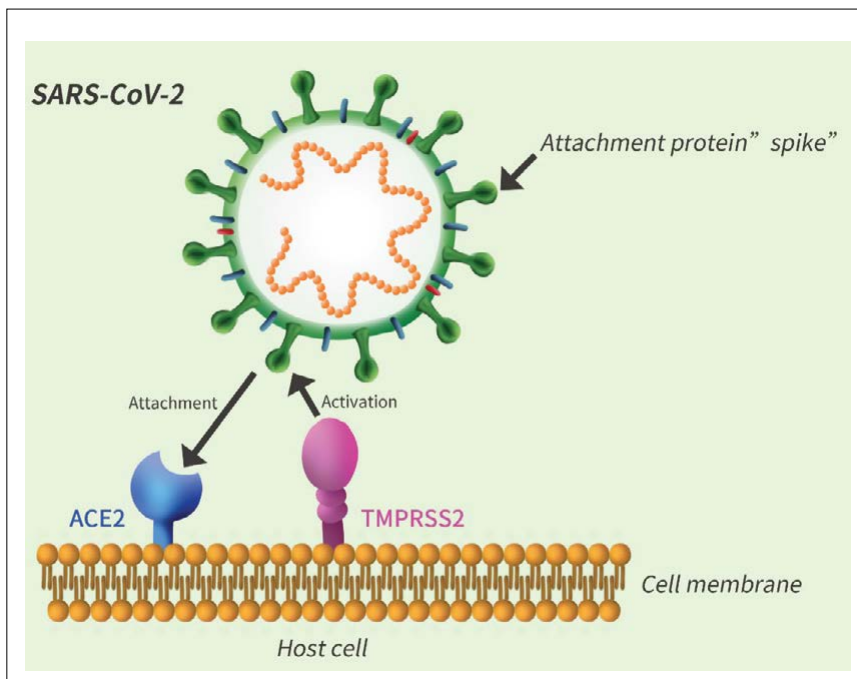

Figure 2: ACE2 and TMPRSS2 in Corona virus

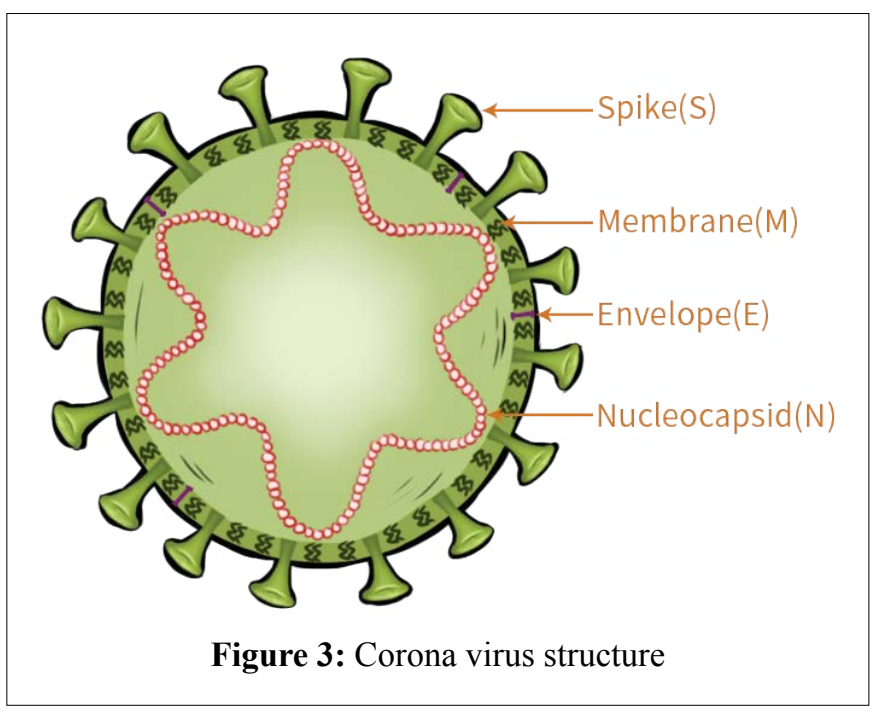

Nucleocapsid: The nucleocapsid phosphoprotein packages the viral genome into a helical ribonucelocapsid, thus playing a crucial role in viral self-assembly (Figure 3).

ACE-2 receptor: ACE-2 is the host cell receptor responsible for mediating infection by SARS-CoV-2 (Figure 4).

Furin: It is a protease present in many human organs that recognizes and activates a specific site on the SARS-CoV-2 Spike protein, thus facilitating a tighter binding to the ACE-2 receptor and might play a role in the higher infection rate [3] (Figure 5).

Cytokines: Studies have shown a strong correlation between severity of the disease and concentrations of IL2, IL7, IL10, GCSF, MCP1 and TNF alpha [1]. Cytokines are a category of small proteins $(\sim 5-20 \mathrm{kDa})$ that is important in cell signaling. Cytokines are peptides and the most important thing is they cannot cross the lipid bilayer of cells to enter

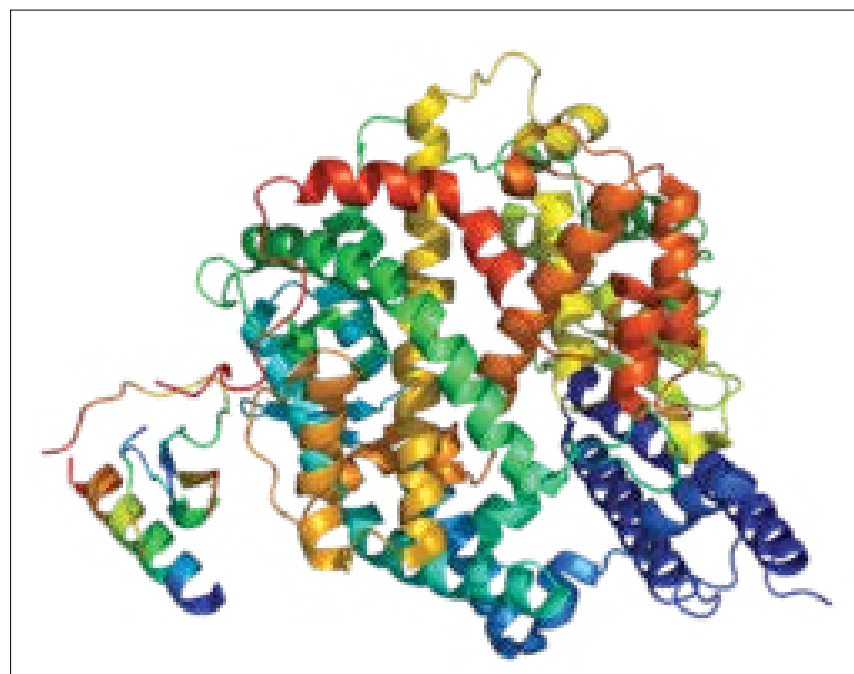

Figure 4: ACE-2 Receptor Protein

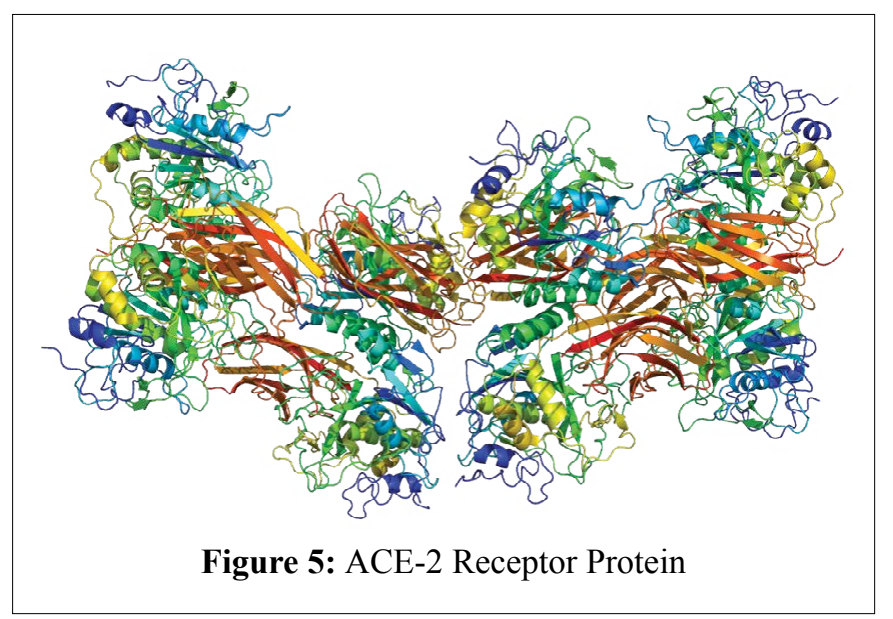

the cytoplasm which means concentrating on Cytokines in COVID-19 is not so important. Cytokines have been shown to be involved in autocrine, paracrine and endocrine signaling as immunomodulation agents. Cytokines include chemokines, interferons, interleukins, lymphokines, and tumor necrosis factors, but generally not hormones or growth factors. Cytokines are produced by a broad range of cells, including immune cells like macrophages, B lymphocytes, $\mathrm{T}$ lymphocytes and mast cells, as well as endothelial cells, fibroblasts, and various stromal cells. A given cytokine may be produced by more than one type of cell. [26-27] They act through cell surface receptors and are especially important in the immune system. cytokines modulate the balance between humoral and cell-based immune responses, and they regulate the maturation, growth, and responsiveness of particular cell populations. Some cytokines enhance or inhibit the action of other cytokines in complex ways. They are different from hormones, which are also important cell signaling molecules. Hormones circulate in higher concentrations, and tend to be made by specific kinds of cells. Cytokines are 
important in health and disease, specifically in host immune responses to infection, inflammation, trauma, sepsis, cancer, and reproduction. In other words, Cytokines are important in immune system functioning and when a pathogen get inside the body, immune related cells distribute these proteins (Figure 6).

\section{DISCUSSIONS}

COVID-19 like Influenza Virus-has 8 RNA segments which can be packaged in different ways when they infect birds, pigs, or humans. [Biology \& Physics at KITP. By Terry Hipsher] Coronaviruses are large pleomorphic spherical particles with bulbous surface projections. [4] The average diameter of the virus particles is around $120 \mathrm{~nm}(.12 \mu \mathrm{m})$. The diameter of the envelope is $\sim 80 \mathrm{~nm}(.08 \mu \mathrm{m})$ and the spikes are $\sim 20 \mathrm{~nm}(.02$ $\mu \mathrm{m})$ long. The envelope of the virus in electron micrographs appears as a distinct pair of electron dense shells. [5-6] The viral envelope consists of a lipid bilayer where the membrane (M), envelope (E) and spike (S) structural proteins are anchored. [7] Inside the envelope, there is the nucleocapsid, which is formed from multiple copies of the nucleocapsid (N) protein, which are bound to the positive-sense singlestranded RNA genome in a continuous beads-on-a-string type conformation. [8-9] The lipid bilayer envelope, membrane proteins, and nucleocapsid protect the virus when it is outside

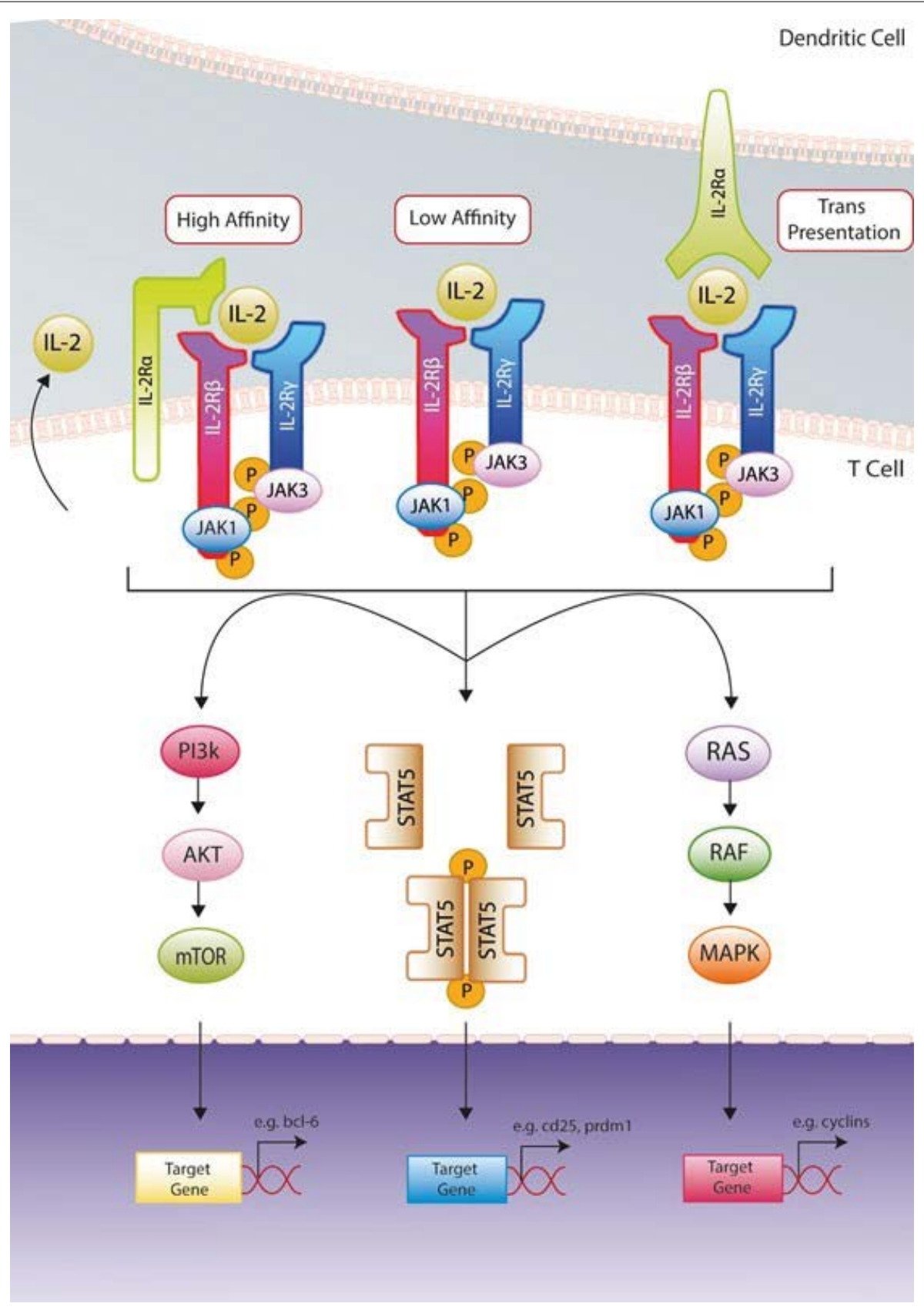

Figure 6: IL2 Pathway 
the host cell. [22] the structure of COVID-19 is so rigid and has very powerful against many antiviral and antimicrobial detergent like alcohol since the outer layer of the virus is lipid and cannot be solved in alcohol based materials.

Based on our calculations, the genome size of COVID-19 as mentioned is from 26 to 32 Kilobases (basepair [bp]). 26 Kilopaepair: 26000 kilobasepair [kb], 0.026 megabasepair $[\mathrm{Mb}], 0.000026$ gigabasepair [Gb], 8666.666667 amino acid [aa], 8666.666667 codon. And 32 Kilobases means: 32000 kilobasepair [kb], 0.032 megabasepair [Mb], 0.000032 gigabasepair [Gb], 10666.666667 amino acid [aa], 10666.666667 codon. As you observe, the codon and aminoacids of COVID-19 are similar which is very rare in Viruses. When the Codon is similar to amino acid number it is called Codon Mismatch which we observe in COVID-19. mitochondria are the targets of the reactive oxygen species (ROS) that are produced inside a cell during viral infections, and that mtDNA is a major target of these ROS [10]. Mitochondrial ATP generation requires proteins from the nuclear and mitochondrial genomes. ROS disrupt the oxidative production of ATP, which is required for normal cellular function, because damage of mtDNA disrupts the normal synthesis of proteins needed for mitochondria function and making them suitable targets for attack by ROS produced during infections by viruses and other microorganisms, although ROS also have other cellular targets. In HIV and hepatitis C virus infections, oxidative stress (OS) always plays a dominant pathogenic role. Peterhen and other researchers showed that almost all viruses (DNA/RNA viruses) cause cell death by generating oxidative stress in infected cells [11-13]. The OS generated during chronic hepatitis is associated with hepatic damage, a decrease in reduced glutathione (GSH) and decrease in plasma and hepatic zinc concentration [1415]. A loss of the MMP leads to imbalances in the membrane potentials of the IM and $\mathrm{OM}$, and then to arrest of normal cellular biosynthetic function and bioenergetics, and finally to a "crisis" within the cell. A loss of the MMP also leads to release of several proapoptotic proteins from the IMS, such as Cyt $\mathrm{C}$ and Smac/DIABLO, as well as caspase independent death effectors, such as apoptosis-inducing factor (AIF) and endonuclease G (EndoG) [16], which have important roles in caspase-independent and caspase-dependent cell death [17]. The MMP transition occurs during the pathogenesis of exogenous factors (e.g., viral proteins, toxins, and prooxidants [18-19]). A prolonged loss of the MMP leads to serious cell damage, from which the cell cannot recover. Therefore, in the intrinsic pathway of apoptosis, any viral factor that influences the MMP has a major impact on cell fate, either by inducing or by blocking cell death [20]. In recent years, there has been an increasing focus on the role of the MMP in disease and health. Thus, several recent models based on in vivo and in vitro studies explain the mechanisms underlying the maintenance and loss of the MMP. A loss of the MMP by any mechanism leads to functional and structural collapse of the mitochondria and cell death [21]. A recent study for first time has shown that dengue virus (DV) infection of human hepatoma cell line (HepG2) leads alteration in the bioenergetic function of mitochondrial morphology leading to MMP loss [22]. The alteration in respiratory properties of HepG2 cells in DV infection results due to decrease in respiratory control ratio (PCR) and $\mathrm{ADP} / \mathrm{O}$ ratio, which suggest significant alteration in mitochondrial morphology. Another additional feature observed by an increase in proton leak termed mitochondrial uncoupling which occurs by leaking of protons through FoF1 ATP synthase from inner membrane into matrix resulting in decrease in MMP loss. Thus, creating an imbalance in ATP synthesis ultimately affects the bioenergetic functions of cell. The biochemical mitochondrial damage induced in cell infected with HCV showed that E1 Protein together with core and NS3 are responsible for ROS production. Core and NS3 induce NO production which causes MMP loss by opening of transition pore [23]. NO could also interact with another free radical superoxide $\left(\mathrm{O}_{2}-\right)$ to form strong peroxynitrite anion (ONOO-), which irreversibly inhibits multiple respiratory complexes (complexes I, II and IV) and aconitase, and activate proton leak and permeability transition pore [24-25]. Therefore, interfering with energy metabolism by disrupting the ATP synthesis of cell results in modulation of mitochondrial function.

\section{CONCLUSION}

Based on this review, a loss of the MMP in mitochondria leads to functional and structural collapse of the mitochondria and cell death. For instance, dengue virus (DV) infection of human hepatoma cell line (HepG2) leads alteration in the bioenergetic function of mitochondrial morphology leading to MMP loss. The alteration in respiratory properties of HepG2 cells in DV infection results due to decrease in respiratory control ratio (PCR) and $\mathrm{ADP} / \mathrm{O}$ ratio, which suggest significant change in mitochondrial morphology. Another additional feature observed by an increase in proton leak termed mitochondrial uncoupling which occurs by leaking of protons through FoF1 ATP synthase from inner membrane into matrix resulting in decrease in MMP loss. Therefore; creating an imbalance in ATP synthesis ultimately affects the bioenergetic functions of cell. The biochemical mitochondrial damage induced in cell infected with HCV showed that E1 Protein together with core and NS3 are responsible for ROS production. Core and NS3 induce NO production which causes MMP loss by opening of transition pore. NO could also interact with another free radical superoxide $(\mathrm{O} 2-)$ to form strong peroxynitrite anion (ONOO), which irreversibly inhibits multiple respiratory complexes (complexes I, II and IV) and aconitase, and activate proton leak and permeability transition pore. Thus, interfering with energy metabolism by disrupting the ATP synthesis of cell results in 
modulation of mitochondrial function. COVID-19 like DV, is related to mitochondrial function and ATP production of the patient. Abnormal Mitochondrial function is an infected patient leads to the abnormal respiratory function and death of the patient. This is the major cause of death of many people infected with SARS-CoV-19.

\section{REFERENCES}

1. Singhal, T. A Review of Coronavirus Disease-2019 (COVID-19). Indian J Pediatr 87, 281-286 (2020). https://doi.org/10.1007/ s12098-020-03263-6.

2. Markus Hoffmann et al, SARS-CoV-2 Cell Entry Depends on ACE2 and TMPRSS2 and Is Blocked by a Clinically Proven Protease Inhibitor, Volume 181, Issue 2, 16 April 2020, Pages 271-280.e8, https://doi.org/10.1016/j.cell.2020.02.052.

3. Daniel Wrapp, Nianshuang Wang, Kizzmekia S. Corbett, Jory A. Goldsmith, Ching-Lin Hsieh, Olubukola Abiona, Barney S. Graham, Jason S. McLellan, Cryo-EM structure of the 2019nCoV spike in the prefusion conformation Science13 Mar 2020: 1260-1263. DOI: 10.1126/science. abb2507.

4. Dr. Royal R. Rife. A Rare Recording of Dr. Royal R. Rife. Release Date: July 20, 2018. Publisher: Listen \& Live Audio. Unabridged Audiobook.

5. Goldsmith CS, Tatti KM, Ksiazek TG, Rollin PE, Comer JA, Lee WW, et al. (February 2004). "Ultrastructural characterization of SARS coronavirus". Emerging Infectious Diseases. 10 (2): 320-26. doi:10.3201/eid1002.030913. PMC 3322934. PMID 15030705 . Virions acquired an envelope by budding into the cisternae and formed mostly spherical, sometimes pleomorphic, particles that averaged $78 \mathrm{~nm}$ in diameter (Figure 1A).

6. Neuman BW, Adair BD, Yoshioka C, Quispe JD, Orca G, Kuhn P, et al. (August 2006). "Supramolecular architecture of severe acute respiratory syndrome coronavirus revealed by electron cryomicroscopy". Journal of Virology. 80 (16): 7918-28. doi:10.1128/JVI.00645-06. PMC 1563832. PMID 16873249. Particle diameters ranged from 50 to $150 \mathrm{~nm}$, excluding the spikes, with mean particle diameters of 82 to $94 \mathrm{~nm}$; Also See Figure 1 for double shell.

7. Fehr AR, Perlman S (2015). Maier HJ, Bickerton E, Britton P (eds.). "Coronaviruses: an overview of their replication and pathogenesis". Methods in Molecular Biology. Springer. 1282: 1-23. doi:10.1007/978-1-4939-2438-7_1. ISBN 978-1-4939-24387. PMC 4369385. PMID 25720466. See section: Virion Structure.

8. Lai MM, Cavanagh D (1997). "The molecular biology of coronaviruses". Advances in Virus Research. 48: 1-100. doi:10.1016/S0065-3527(08)60286-9. ISBN 9780120398485. PMID 9233431.

9. Chang CK, Hou MH, Chang CF, Hsiao CD, Huang TH (March 2014). "The SARS coronavirus nucleocapsid protein-forms and functions". Antiviral Research. 103: 39-50. doi: 10.1016/j. antiviral.2013.12.009. PMID 24418573. See Figure 4c.

10. Neuman BW, Kiss G, Kunding AH, Bhella D, Baksh MF, Connelly S, et al. (April 2011). "A structural analysis of M protein in coronavirus assembly and morphology". Journal of Structural Biology. 174 (1): 11-22. doi: 10.1016/j.jsb.2010.11.021. PMC 4486061. PMID 21130884. See Figure 10.
11. Reshi L, Wang HV, Hui CF, Su YC, Hong JR. Anti-apoptotic genes Bcl-2 and Bcl-xL overexpression can block iridovirus serine/threonine kinase-induced Bax/mitochondria-mediated cell death in GF-1 cells. Fish \& Shellfish Immunology. 2017; 61:120129.

12. Peterhans E, Grob M, Urge TB, Zanoni R. Virus-induced formation of reactive oxygen intermediates in phagocytic cells. Free Radical Research Communications. 1987;3(1-5):39-46.

13. Vierucci A, DeMartino M, Graziani E. A mechanism for liver cell injury in viral hepatitis: Effects of hepatitis B virus on neutrophil function in vitro and in children with chronic active hepatitis. Pediatric Research. 1983;17(10):814-820.

14. Muller F. Reactive oxygen intermediates and human immunodeficiency virus (HIV) infection. Free Radical Biology and Medicine. 1992;13(6):651-657.

15. Boya P, Peña AD, Beloquietal O. Antioxidant status and glutathione metabolism in peripheral blood mononuclear cells from patients with chronic hepatitis C. Journal of Hepatology. 1999;31(5):808-814.

16. Bianchi GP, Marchesini G, Brizietal M. Nutritional effects of oral zinc supplementation in cirrhosis. Nutrition Research. 2000;20(8):1079-1089.

17. Reshi L, Su YC, Hong JR. RNA viruses: ROS-mediated cell death. International Journal of Cell Biology. 2014; 2014:467-452.

18. Reshi L, Wu JL, Wang HV, Hong JR. Aquatic viruses induce host cell death pathways and its application. Virus Research. 2016; 211:133-144.

19. Reshi L, Hong JR. Mitochondria as a favorite organelle for invading viruses. Molecular Biology. 2017; 6:181.

20. Reshi L, Su YC, Hong JR. RNA viruses: ROS-mediated cell death. International Journal of Cell Biology. 2014; 2014:467-452.

21. Machida $\mathrm{K}$ et al. Hepatitis $\mathrm{C}$ virus infection activates the immunologic (type II) isoform of nitric oxide synthase and thereby enhances DNA damage and mutations of cellular genes. Journal of Virology. 2004;78(16):8835-8843.

22. Reshi L, Wu JL, Wang HV, Hong JR. Aquatic viruses induce host cell death pathways and its application. Virus Research. 2016; 211:133-144.

23. Machida $\mathrm{K}$ Et al. Hepatitis $\mathrm{C}$ virus infection activates the immunologic (type II) isoform of nitric oxide synthase and thereby enhances DNA damage and mutations of cellular genes. Journal of Virology. 2004;78(16):8835-8843.

24. Reshi L, Hong JR. Mitochondria as a favourite organelle for invading viruses. Molecular Biology. 2017; 6:181.

25. Meng G, Xia M, Wang D, Chen A, Wang Y, Wang H, Yu D, Wei J. Mitophagy promotes replication of oncolytic Newcastle disease virus by blocking intrinsic apoptosis in lung cancer cells. Oncotarget. 2014;5(15):6365-6374.

26. "Cytokine" in John Lackie. A Dictionary of Biomedicine. Oxford University Press. 2010. ISBN 9780199549351.

27. "Cytokine" in Stedman's Medical Dictionary, 28th ed. Wolters Kluwer Health, Lippincott, Williams \& Wilkins (2006). 